\title{
Psicooncología
}

ISSN: $1696-7240$

\section{Ansiedad y depresión en hombres con cáncer de próstata en función del tipo de tratamiento y su relación con la calidad de vida y la información recibida}

\author{
Natalia Oraá-Tabernero ${ }^{1, *}$; Juan Antonio Cruzado ${ }^{2}$
}

Recibido: 1 de agosto de 2019 / Aceptado: 5 de septiembre de 2019

Resumen: Objetivo: Conocer el nivel de ansiedad y depresión que tienen los pacientes con cáncer de próstata en función de variables sociodemográficas (edad, nivel de estudios, situación laboral o tener pareja) y variables clínicas (grupo de riesgo, tipo de tratamiento o fase de supervivencia) y cómo el estado emocional puede variar en función de la calidad de vida y la información recibida. Método: Se seleccionaron aleatoriamente 176 hombres, entre 2015-2016, que habían recibido tratamientos locales, hormonales o combinados. Se realizó una entrevista y se administró los cuestionarios BAI, BDI-II, EORTC QLQ-C30, EORTC QLQPR25 y EORTC QLQ-INFO25. Resultados: Las puntuaciones de los inventarios BAI y BDI-II son bajas, los síntomas de depresión son más elevados y aparecen con más frecuencia. No existen diferencias significativas en función de las variables sociodemográficas ni tampoco en función de variables clínicas como el grupo de riesgo o la fase de supervivencia. Sin embargo, los hombres que reciben tratamientos hormonales padecen más ansiedad y depresión que aquellos que reciben tratamientos locales. Además, los hombres, que tienen peor calidad de vida y/o recibieron peor información, presentan más síntomas de ansiedad y depresión. Conclusiones: En general, nuestros hombres no presentan ansiedad o depresión clínica. Se debe prestar especial cuidado a los hombres que reciben tratamientos hormonales, aquellos que padecen efectos adversos derivados de los tratamientos o presentan peor calidad de vida. Y en todos los casos, es necesario mejorar la comunicación desde el momento del diagnóstico, entre otras cosas, porque el hombre informado adecuadamente presenta mejor estado emocional.

Palabras clave: Cáncer de próstata, tratamiento local, tratamiento sistémico, ansiedad, depresión, calidad de vida, información.

\section{[en] Anxiety and depression in men with prostate cancer depending on the type of treatment and its relationship with the quality of life and the information received}

\footnotetext{
Abstract: Objective: To know the level of anxiety and depression that patients with prostate cancer have based on sociodemographic variables (age, level of studies, employment status or having a partner) and clinical variables (risk group, type of treatment or survival phase), and how the emotional

1 Natalia Oraá-Tabernero, Centro de Psico-Oncología, Vara de Rey 9-3ํ․ Logroño.

E-mail: noraa@cop.es

2 Juan A. Cruzado-Rodríguez, Facultad de Psicología, Universidad Complutense de Madrid. Madrid.

E-mail: jacruzad@ucm.es

Declaración: Todos los autores cumplen los requisitos de autoría y declaran la no existencia de conflictos de intereses en este estudio.

* Dirección de Correspondencia: Natalia Oraá Tabernero. Centro de Psico-Oncología. Vara de Rey 9-3E. 26001Logroño (La Rioja). España. E-mail: info@nataliaoraa.es
} 
state can vary depending on the quality of life and the information received. Method: 176 men were randomly selected, between 2015-2016, who had received local, hormonal or combined treatments. An interview was conducted and the BAI, BDI-II, EORTC QLQ-C30, EORTC QLQ-PR25 and EORTC QLQ-INFO25 questionnaires were administered. Results: BAI and BDI-II inventory scores are low, depression symptoms are higher and appear more frequently. There are no significant differences depending on the sociodemographic variables or clinical variables such as the risk group or the survival phase. However, men who receive hormonal treatments suffer more anxiety and depression than those who receive local treatments. In addition, men who have worse quality of life and / or received worse information have more symptoms of anxiety and depression. Conclusions: In general, our men do not present anxiety or clinical depression. Special care should be given to men who receive hormonal treatments, those who suffer adverse effects from the treatments or have a worse quality of life. And in all cases, it is necessary to improve communication from the moment of diagnosis, among other things, because the properly informed man has a better emotional state.

Keywords: Prostate cancer, local treatment, systemic treatment, anxiety, depression, quality of life, information.

Sumario. 1. Introducción 2. Método 3. Análisis de datos 4. Resultados

Cómo citar: Oraá-Tabernero N, Cruzado JA. Ansiedad y depresión en hombres con cáncer de próstata en función del tipo de tratamiento y su relación con la calidad de vida y la información recibida. Psicooncología 2019; 16: 329-344. doi: 10.5209/psic.65594

\section{Introducción}

El incremento de la población y su envejecimiento influyen, entre otros factores, en que el cáncer de próstata sea uno de los tumores de mayor incidencia. Así, según la Sociedad Española de Oncología Médica en España en 2019 uno de los diagnósticos oncológicos más frecuentes en la población masculina será el cáncer de próstata ${ }^{(1)}$. Un diagnóstico que, por otro lado, supone en el hombre un impacto importante que afecta a su funcionamiento físico, psicológico y socio-familiar ${ }^{(2,3)}$ constituyendo, dada su prevalencia y su impacto, uno de los principales problemas sanitarios que existen hoy en día.

La etiología de padecer ansiedad o depresión, a la hora de afrontar un cáncer de próstata, está muy relacionada con variables clínicas como la fase de tratamiento (diagnóstico, tratamiento activo, supervivencia o recidiva) y el tipo de tratamiento recibido con sus posibles efectos adversos. En cuanto a la fase del tratamiento en la que se encuentre la persona, el malestar emocional aparece con más frecuencia cuando se realizan las pruebas para descartar el tumor como la del antígeno prostático específico (PSA), en el diagnóstico, en la toma de decisiones de las diferentes opciones terapéuticas, durante la administración del tratamiento o en el momento de la recidiva ${ }^{(4)}$. Un reciente meta-análisis sobre los trastornos de ansiedad y depresión en estos hombres concluyó que la prevalencia de ambos trastornos eran más altas entre los pacientes cuando no habían comenzado el tratamiento ( $27,40 \%$ y $17,27 \%$, respectivamente) las más bajas cuando se encontraban durante el tratamiento activo $(15,90 \%$ y $14,70 \%$, respectivamente), y volvían a aumentar una vez terminaban dicho tratamiento $(18,49 \% \text { y } 18,44 \% \text {, respectivamente })^{(5)}$, pudiendo incluso llegar a cuadriplicarse la tasa de suicidio en los hombres con cáncer de próstata en comparación con sus iguales en la población general ${ }^{(6)}$. Por otra parte, los 
pacientes con esta enfermedad que además padecen trastornos del estado del ánimo, necesitan un mayor uso de los recursos sanitarios, ya que acuden con más asiduidad al médico de atención primaria, a urgencias, requieren de más hospitalizaciones, medicamentos y pruebas adicionales, lo que incide directamente en un mayor gasto sanitario ${ }^{(7)}$. Con respecto al tipo de tratamiento, hoy en día existen diferentes opciones cuya alternativa dependerá de diferentes factores como el grupo de riesgo. El grupo de riesgo es un factor pronóstico específico del diagnóstico del cáncer de próstata que ayuda a estimar el riesgo de recurrencia bioquímica y por lo tanto predecir su eficacia. El grupo de riesgo es uno de los factores más utilizados y viene determinado por los resultados obtenidos en otras pruebas diagnósticas como PSA, la puntuación de Gleason y la etapa de clasificación T para dividir a los hombres en diferentes grupos: bajo, intermedio, alto y avanzado; cuanto más bajo es el grupo de riesgo, más baja es la probabilidad de que el cáncer crezca y se disemine ${ }^{(8,9)}$. Entre los diferentes tratamientos disponibles encontramos los tratamientos locales, cuyo objetivo es eliminar el cáncer de un área específica y limitada (cirugía o radioterapia, principalmente), los tratamientos sistémicos cuyo objetivo es distribuir el tratamiento por todo el cuerpo (terapias hormonales o quimioterapia, principalmente) y los tratamientos combinados en los que se aplica terapia de privación androgénica (ADT) adyuvante a un tratamiento local.

Cuando se evalúa el estado emocional de estos hombres, se observa que tienden a sufrir ansiedad y/o depresión cuando el tratamiento local ha producido efectos adversos relacionados principalmente con la incontinencia urinaria, las disfunciones sexuales y los problemas intestinales o bien, si con el tratamiento sistémico, se está sufriendo fatiga, disnea, insomnio, dolor o, como ocurría en los tratamientos locales, disfunciones sexuales ${ }^{(4,10,11)}$, llegando incluso a reducir significativamente la calidad de vida en todas sus áreas (física, de rol, emocional, cognitiva y social) y minando su bienestar emocional y su vida personal, familiar y social ${ }^{(12,13)}$.

Algo que debiera ser preocupante para los profesionales de este campo es el hecho de que, a pesar de lo expuesto anteriormente, los servicios de apoyo que están disponibles para los hombres con cáncer de próstata, a la hora de tomar decisiones, manejar la ansiedad o la depresión, son muy escasos y un elevado número de pacientes con cáncer de próstata que presentan dificultades emocionales no son tratados psicológicamente, bien porque los sistemas de salud carecen de medios para su identificación y tratamiento o bien porque los pacientes o sus cuidadores no lo solicitan explícitamente ${ }^{(14,15)}$. Para prevenir que esto siga ocurriendo, es necesario que seamos conscientes de la importancia que tiene la comunicación en la relación médico-paciente, entre otras cosas porque facilitar adecuadamente la información necesaria influye en que el paciente se adapte mejor a los tratamientos, aumente la adherencia y mejoren las habilidades personales para afrontar la enfermedad ${ }^{(16)}$, siendo más fácil detectar, por parte del facultativo, las diferentes necesidades que pueda tener su paciente o que éste tenga la confianza necesaria con su médico para solicitar ayuda.

Elobjetivo principal del presente estudio es conocer el estado de ansiedad ydepresión en los hombres con cáncer de próstata y las diferencias que existen en la ansiedad (BAI) y la depresión (BDI-II) en función de diferentes variables sociodemográficas (edad, nivel de estudios, situación laboral o tener pareja) y variables clínicas (grupo de riesgo, tipo de tratamiento o fase de supervivencia) y cómo la calidad de vida y la información recibida pueden influir en su estado emocional. 


\section{Método}

\section{Muestra}

De los hombres diagnosticados de cáncer de próstata entre 2013- 2015, se invitó a participar a 216 hombres que estaban siendo atendidos bien por el Servicio de Radioterapia Oncológica de la Fundación Rioja Salud, bien por el Servicio de Urología del Hospital San Pedro de Logroño. En el momento de la entrevista, estaban recibiendo tratamiento activo sistémico (concretamente ADT) desde hacía un mínimo de 3 meses o habían sido intervenidos de un tratamiento local como mínimo 3 meses antes.

Para ser incluidos en el estudio era necesario que obtuvieran una puntuación de Karnofsky $>80$ o Mini Mental $>26$ puntos ${ }^{(17,18)}$, además de entender castellano, no encontrarse en una situación paliativa ni tener antecedentes de trastornos mentales. Finalmente, se evaluó a 176 pacientes $(81,48 \%)$, excluyendo a $40(18,51 \%)$ por motivos como rehusar a colaborar $(\mathrm{n}=23)$, no cumplir los criterios de selección $(n=17)$ u otros motivos $(n=5)$.

Se trata de un estudio observacional, descriptivo, trasversal, de elección aleatoria de los hombres con cáncer de próstata.

\section{Variables e Instrumentos}

Los hombres se agruparon en 4 grupos en función del grupo de riesgo (bajo, intermedio, alto y avanzado). Por otro lado, se dividieron en 3 grupos en función del tipo de tratamiento: local, combinado o sistémico (terapia hormonal, ADT o supresora de andrógenos). Por último, se les agrupó en 3 grupos en función de la fase de supervivencia (3-12 meses, 12-24 meses o 24-36 meses).

Como instrumentos de selección se utilizaron la Escala Karnofsky que mide la capacidad funcional ${ }^{(17)}$ y el Mini Examen Cognoscitivo que comprueba el deterioro cognitivo ${ }^{(18,19)}$.

La variable "ansiedad" se evaluó basándonos en los resultados obtenidos en la aplicación del Inventario de Ansiedad de Beck (BAI) ${ }^{(20-22)}$ y la variable "depresión" con los resultados del Inventario de Depresión de Beck- II (BDI-II) ${ }^{(23)}$.

Para evaluar la calidad de vida y la calidad de la información recibida se utilizaron los cuestionarios y los módulos de la EORTC, cuyas puntuaciones se transforman en una puntuación lineal en escala de 0-100 de acuerdo con el manual de la EORTC $(24,25)$.

La calidad de vida general se evaluó a través del cuestionario EORTC QLQ-C30, la calidad de vida en el cáncer de próstata con el módulo EORTC QLQ-PR25 y para valorar la calidad de la información recibida se utilizó el módulo EORTC QLQ-INFO25. Han demostrado ser válidos y fiables para muestras tanto internacionales como españolas ${ }^{(26-31)}$.

\section{Procedimiento}

Tras explicar los objetivos del estudio y firmar el consentimiento informado se procedía a la entrevista, con el fin de recoger los datos sociodemográficos y clínicos para posteriormente aplicar los cuestionarios: BAI, BDI-II, EORTC QLQ-C30, EORTC QLQ-PR25 y el EORTC QLQ-INFO25(20,23,28-30).

El Comité Ético de Investigación Clínica de La Rioja dictaminó que el estudio cumplía los aspectos metodológicos, éticos y legales que permitían su realización (CEICLAR P.I. $n^{\circ} 65$ del 08 de julio del 2015; Ref. CEICLAR PI-192). 


\section{Análisis de datos}

Se realizó el análisis descriptivo de todas las variables, bien mediante las frecuencias absolutas y relativas de las variables cualitativas (sociodemográficas y clínicas), bien mediante el análisis estadístico, medias y desviaciones típicas del resto de las variables cuantitativas (BAI, BDI-II, EORTC QLQ-C30, EORTCQLQ-PR25 y EORTC QLQ-INFO25).

Se calculó la correlación de Pearson entre la ansiedad (BAI) y depresión (BDI-II) y las variables cuantitativas edad, calidad de vida general y específica de cáncer de próstata (EORTC QLQ-C30 y PR25) e información (EORTC QLQ-INFO25).

Para comprobar las diferencias según el nivel de estudio, situación laboral y las variables clínicas grupo de riesgo y tratamiento en la ansiedad y depresión de estos hombres, se llevó a cabo la prueba de Kruskall-Wallis con prueba post hoc de contraste Dunn-Bonferroni y se calculó el tamaño del efecto mediante la d de Cohen. En cuanto a las diferencias en la ansiedad y depresión, en función tener o no pareja, se llevó a cabo una prueba t de Student. Para terminar, se realizó un ANOVA para evaluar las posibles diferencias en la ansiedad y depresión, en función de la fase de supervivencia en la que se encontrara el sujeto.

El análisis de datos se realizó con el paquete estadístico SPSS versión 22. Un valor asociado $\mathrm{p}<0,05$ fue considerado como estadísticamente significativo.

\section{Resultados}

La mayoría de los sujetos tenían edades superiores a 60 años, con estudios básicos, jubilados y con pareja; lo más frecuente fue tener en el momento de diagnóstico el grupo de riesgo bajo y encontrarse en seguimiento de 24-36 meses. Los tratamientos más habituales fueron los tratamientos locales seguidos del combinado (tabla 1).

Tabla 1. Características demográficas y clínicas de la muestra

\begin{tabular}{lcc}
\hline VARIABLES & & \\
\hline EDAD (media y desviación típica) & $68,32(7,58)$ & \\
& $\underline{\boldsymbol{N}}$ & $\underline{\mathbf{o}}$ \\
NIVEL ACADÉMICO & 110 & 62,5 \\
Básicos & 41 & 23,3 \\
Secundarios & 25 & 14,2 \\
Superiores & & \\
SITUACIÓN LABORAL & 134 & 76,1 \\
Jubilado & 31 & 17,6 \\
En activo & 11 & 6,3 \\
Baja Laboral & & \\
PAREJA & 144 & 81,8 \\
Con pareja & 35 & 18,2 \\
Sin pareja &
\end{tabular}




\begin{tabular}{lcc}
\hline VARIABLES & & \\
\hline GRUPO DE RIESGO & 70 & 39,8 \\
Bajo & 53 & 30,1 \\
Intermedio & 45 & 25,6 \\
Alto & 8 & 4,5 \\
Avanzado & & \\
TIPO DE TRATAMIENTO & 85 & 48,29 \\
Local & 64 & 36,36 \\
Combinado & 27 & 15,34 \\
Sistémico & & \\
FASE SUPERVIVENCIA & 47 & 26,7 \\
3-12 meses & 47 & 26,7 \\
12-24 meses & 82 & 46,6 \\
24-36 meses & & \\
\hline
\end{tabular}

En la tabla 2 se describen los datos de la muestra con relación a los inventarios BAI y BDI-II. De los 176 sujetos analizados el 2,84\% presentaron ansiedad clínica y el 11,36\% depresión, siendo el trastorno depresivo el más frecuente. Además, todos los hombres que padecían ansiedad clínica tenían asociado un trastorno depresivo. Por otro lado, las puntuaciones totales en ambos cuestionarios son bajas, siendo la puntuación máxima obtenida y la media global en el inventario de depresión superior a las obtenidas en el inventario de ansiedad (41 vs. 35 y 7,85 vs. 6,34, respectivamente).

Tabla 2. Media y Desviaciones Típicas de los inventarios BAI y BDI-II

\begin{tabular}{cccc}
\hline VARIABLES & Punto de corte & N & \% \\
\hline Ansiedad & 21 & 5 & $2,84 \%$ \\
Depresión & 21 & 20 & $11,36 \%$ \\
\hline INVENTARIOS & Media (DT) & Rango & Intervalo de confianza (95\%) \\
\hline BAI & $6,34(6,30)$ & $0-35$ & $5,40-7,27$ \\
BDI-II & $7,85(8,56)$ & $0-41$ & $6,57-9,12$ \\
\hline
\end{tabular}

BAI: Inventario de Ansiedad de Beck; BDI-II: Inventario de Depresión de Beck-II. DT: Desviación Típica.

En la tabla 3 se exponen los datos para la muestra total en calidad de vida, general y específica, y en la información recibida. Los resultados de la muestra total indican que la puntuación global de la calidad de vida general es satisfactoria, tanto en las escalas funcionales como en la global de salud. De las escalas funcionales, la escala emocional es la más baja. La sintomatología específica del cáncer de próstata más afectada es el insomnio, la actividad sexual y los síntomas urinarios, aunque en general son bajas. 
En cuanto a la información recibida, tanto la puntuación global como las diferentes escalas, son medias-bajas a excepción de la escala "información sobre otros servicios" y los ítems "otros lugares de cuidado", "pautas de autocuidado" e "información en CD" que son muy bajos. En cuanto a la satisfacción y la utilidad de la información, se obtienen puntuaciones más o menos satisfactorias.

Tabla 3. Media y desviaciones típicas de los Cuestionarios EORTC QLQ C30, PR25 e INFO25

\begin{tabular}{|c|c|c|c|}
\hline EORTC QLQ-C30 & Media & $\begin{array}{l}\text { Desviación } \\
\text { Típica }\end{array}$ & $\begin{array}{c}\text { Intervalo de confianza } \\
\text { para la media }(95 \%)\end{array}$ \\
\hline Escala global de salud & 76,75 & 17,26 & $74,18-79,32$ \\
\hline \multicolumn{4}{|l|}{ Escalas Funcionales } \\
\hline Funcionamiento físico & 88,44 & 18,68 & $85,66-91,22$ \\
\hline Funcionamiento de rol & 86,55 & 28,26 & $82,34-90,75$ \\
\hline Funcionamiento emocional & 77,74 & 25,10 & $74,01-81,48$ \\
\hline Funcionamiento cognitivo & 80,58 & 26,85 & $76,59-84,58$ \\
\hline Funcionamiento social & 89,96 & 21,53 & $86,75-93,16$ \\
\hline \multicolumn{4}{|l|}{ Escala síntomas } \\
\hline Fatiga & 14,45 & 22,42 & $11,12-17,79$ \\
\hline Nauseas & 1,42 & 7,31 & $0,33-2,50$ \\
\hline Dolor & 9,65 & 18,20 & $6,95-12,36$ \\
\hline Disnea & 5,68 & 16,11 & $3,28-8,07$ \\
\hline Insomnio & 23,67 & 33,82 & $18,64-28,70$ \\
\hline Pérdida de apetito & 5,11 & 16,87 & $2,60-7,62$ \\
\hline Estreñimiento & 15,15 & 28,46 & $10,91-19,38$ \\
\hline Diarrea & 10,60 & 24,19 & $7,00-14,20$ \\
\hline Dificultades financieras & 1,89 & 10,52 & $0,32-3,45$ \\
\hline EORTC QLQ-PR25 & Media & $\begin{array}{l}\text { Desviación } \\
\text { Típica }\end{array}$ & $\begin{array}{c}\text { Intervalo de confianza } \\
\text { para la media }(95 \%)\end{array}$ \\
\hline \multicolumn{4}{|l|}{ Escala Síntomas } \\
\hline Urinarios & 22,70 & 17,19 & $20,14-25,26$ \\
\hline Intestinales & 4,16 & 8,35 & $2,92-5,40$ \\
\hline Hormonales & 12,97 & 11,81 & $11,21-14,73$ \\
\hline Actividad sexual & 26,13 & 29,68 & $21,70-30,55$ \\
\hline Funcionamiento sexual & 42,93 & 24,27 & $35,72-50,14$ \\
\hline Uso de prenda urinaria & 22,22 & 34,27 & $9,42-35,02$ \\
\hline EORTC QLQ-INFO25 & Media & $\begin{array}{l}\text { Desviación } \\
\text { Típica }\end{array}$ & $\begin{array}{c}\text { Intervalo de confianza } \\
\text { para la media }(95 \%)\end{array}$ \\
\hline Puntuación global & 43,03 & 9,69 & $41,59-44,47$ \\
\hline \multicolumn{4}{|l|}{ Escalas } \\
\hline Información sobre enfermedad & 53,88 & 23,93 & $50,32-57,44$ \\
\hline Información sobre pruebas médicas & 66,41 & 26,71 & $62,43-70,38$ \\
\hline
\end{tabular}




\begin{tabular}{|c|c|c|c|}
\hline EORTC QLQ-INFO25 & Media & $\begin{array}{l}\text { Desviación } \\
\text { Típica }\end{array}$ & $\begin{array}{c}\text { Intervalo de confianza } \\
\text { para la media }(95 \%)\end{array}$ \\
\hline Información sobre tratamientos & 61,96 & 27,92 & $57,80-66,11$ \\
\hline Información sobre otros servicios & 7,67 & 12,48 & $5,81-9,52$ \\
\hline \multicolumn{4}{|l|}{ Ítems individuales } \\
\hline Información sobre lugares de cuidado & 3,97 & 14,36 & $1,84-6,11$ \\
\hline Información pautas autocuidado & 13,25 & 23,11 & $9,81-16,69$ \\
\hline Información escrita & 55,68 & 49,81 & $48,27-63,09$ \\
\hline Información cd & 1,13 & 10,62 & $-0,44-2,71$ \\
\hline Satisfacción información & 73,86 & 27,34 & $66,79-77,93$ \\
\hline Recibir más información & 17,61 & 38,20 & $11,93-23,29$ \\
\hline Recibir menos información & 1,70 & 12,98 & $-0,22-3,63$ \\
\hline Utilidad de la información & 72,91 & 28,15 & $68,72-77,10$ \\
\hline
\end{tabular}

En las variables sociodemográficas se observa que no existen diferencias significativas ni en la ansiedad ni en la depresión en función de la edad ( $\mathrm{r}=-0,01$, $\mathrm{p}=0,952$ y $\mathrm{r}=0,03, \mathrm{p}=0,635$; respectivamente), el nivel de estudios $(\mathrm{p}=0,836$ y $\mathrm{p}=0,396$; respectivamente), la situación laboral $(\mathrm{p}=0,681 \mathrm{y} \mathrm{p}=0,399$; respectivamente) ni tener pareja ( $\mathrm{t}=0,56, \mathrm{p}=0,572$ y $\mathrm{t}=1,04, \mathrm{p}=0,296$; respectivamente).

Con respecto a las variables clínicas, ni en la ansiedad y ni en la depresión existen diferencias significativas en función del grupo de riesgo en el que se encuentre el sujeto en el momento del diagnóstico ( $p=0,260$ y $p=0,110$; respectivamente), ni en función de la fase de supervivencia ( $\mathrm{p}=0,403 \mathrm{y} \mathrm{p}=0,976$; respectivamente).

Sin embargo, sí existen diferencias significativas en relación con el tipo de tratamiento que esté recibiendo el sujeto. Así, los hombres que se encuentran en tratamientos hormonales padecen más niveles de ansiedad y depresión en comparación con quienes han sido intervenidos con tratamientos locales (tabla 4). No existen diferencias significativas en los hombres que son tratados con tratamientos combinados con respecto a los tratamientos locales o los sistémicos.

Tabla 4. Ansiedad (BAI) y depresión (BDI-II) en función del tratamiento recibido, resultado de la prueba de Kruskall-Wallis y d de Cohen

\begin{tabular}{|c|c|c|c|c|c|c|}
\hline \multirow[t]{3}{*}{ INVENTARIOS } & \multicolumn{3}{|c|}{ TRATAMIENTO } & \multicolumn{3}{|c|}{ ESTADÍSTICOS } \\
\hline & $\begin{array}{r}\text { Local } \\
(\mathrm{n}=85)\end{array}$ & $\begin{array}{c}\text { Combinado } \\
(\mathrm{n}=64)\end{array}$ & $\begin{array}{c}\text { Sistémico } \\
(\mathrm{n}=27)\end{array}$ & & & \\
\hline & $\begin{array}{l}\text { Media } \\
(\text { DT })^{a}\end{array}$ & Media (DT) & $\begin{array}{c}\text { Media } \\
\text { (DT) }\end{array}$ & $\begin{array}{l}\text { K-W } \\
(p)^{b}\end{array}$ & $d^{\mathrm{c}}$ & $\begin{array}{c}\text { Dunn-Bonferroni } \\
\text { (p) }\end{array}$ \\
\hline $\mathbf{B A I} \mathbf{I}^{\mathrm{d}}$ & $5,38(5,93)$ & $6,22(5,01)$ & $9,63(8,85)$ & 0,028 & $\begin{array}{c}\text { L-S } \\
(-0,63)\end{array}$ & 0,038 \\
\hline BDI-II $^{\mathrm{e}}$ & $6,73(8,25)$ & $7,27(6,86)$ & $\begin{array}{c}12,74 \\
(11,38)\end{array}$ & 0,023 & $\begin{array}{c}\text { L-S } \\
(-0,66)\end{array}$ & 0,019 \\
\hline
\end{tabular}

${ }^{a}$ DT: Desviación Típica. ${ }^{b} \mathrm{~K}-\mathrm{W}$ : Kruskal- Wallis. ${ }^{\mathrm{c} d}$ : Índice de Cohen. ${ }^{\mathrm{d} B A I}$ : Inventario de

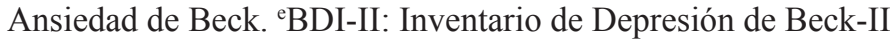


En la tabla 5 se pueden observar los resultados de las asociaciones existentes entre la ansiedad, la depresión y la calidad de vida de estos sujetos. Según los resultados, los hombres que padecen más ansiedad son aquellos que peor calidad de vida presentan, además de tener peor estado global de salud y funcionamiento físico, de rol, emocional, cognitivo y social y en la sintomatología padecen más fatiga, dolor, insomnio, diarrea y dificultades económicas y más efectos adversos urinarios y hormonales.

De manera similar, aquellos hombres que se encuentran más deprimidos puntúan peor en la escala global de calidad de vida, y en las funcionales física, de rol, emocional, cognitiva y social. Así mismo, padecen más fatiga, dolor y dificultades económicas y, en cuanto a la sintomatología específica del cáncer de próstata, los sujetos más deprimidos presentan más problemas urinarios, intestinales y hormonales.

Tabla 5. Matriz de correlaciones entre las puntuaciones de los inventarios de ansiedad y depresión (BAI Y BDI-II) y de las puntuaciones de calidad de vida (EORTC QLQ-C30 y PR25)

\begin{tabular}{lcc}
\hline \multicolumn{1}{c}{ EORTC QLQ-C30 } & BAI ${ }^{\text {a }}$ & BDI-II ${ }^{\mathrm{b}}$ \\
\hline Escala global de salud & $-0,41^{* *}$ & $-0,54^{* *}$ \\
Escalas Funcionales & & \\
Funcionamiento físico & $-0,36^{* *}$ & $-0,45^{* *}$ \\
Funcionamiento de rol & $-0,40^{* *}$ & $-0,54^{* *}$ \\
Funcionamiento emocional & $-0,60^{* *}$ & $-0,56^{* *}$ \\
Funcionamiento cognitivo & $-0,37^{* *}$ & $-0,40^{* *}$ \\
Funcionamiento social & $-0,38^{* *}$ & $-0,45^{* *}$ \\
\cline { 1 - 2 } Escala síntomas & & \\
Fatiga & $0,41^{* *}$ & $0,50^{* *}$ \\
Nauseas & 0,05 & $-0,04$ \\
Dolor & $0,25^{* *}$ & $0,24^{* *}$ \\
Disnea & 0,13 & 0,10 \\
Insomnio & $0,23^{* *}$ & $0,19^{*}$ \\
Pérdida de apetito & 0,09 & 0,13 \\
Estreñimiento & 0,06 & 0,09 \\
Diarrea & $0,24^{* *}$ & $0,16^{*}$ \\
Dificultades financieras & $0,21^{* *}$ & $0,26^{* *}$ \\
\multicolumn{1}{c}{ EORTC QLQ-PR25 } & & \\
Escala Síntomas & & $0,45^{* *}$ \\
Urinarios & $0,39^{* *}$ & $0,23^{* *}$ \\
Intestinales & $0,15^{*}$ & $0,31^{* *}$ \\
Hormonales & $0,25^{* *}$ & $-0,17^{*}$ \\
Actividad sexual & $-0,08$ & 0,01 \\
Funcionamiento sexual & 0,13 & 0,17 \\
Uso de prenda urinaria & 0,01 & \\
\hline & &
\end{tabular}

${ }^{\mathrm{a}}$ BAI: Inventario de Ansiedad de Beck ${ }^{\mathrm{b}}$ BDI-II: Inventario de Depresión de Beck-II ${ }^{*}$ Correlación significativa en el nivel $0,05(2$ colas $){ }^{* *}$ Correlación significativa en el nivel $0,01$ ( 2 colas $)$ 
Para terminar, los resultados de la correlación de Pearson entre la ansiedad y depresión y la información recibida, con asociaciones significativas $\geq 0,20$, indican que los hombres que padecen menos niveles de ansiedad y depresión han recibido más información sobre los tratamientos, están más satisfechos, consideran de mayor utilidad la información recibida y no desean recibir más información (tabla 6).

Tabla 6. Matriz de correlaciones entre las puntuaciones de los inventarios de ansiedad y depresión (BAI Y BDI-II) y del cuestionario de información (EORTC QLQ-INFO25)

\begin{tabular}{lll}
\hline \multicolumn{1}{c}{ EORTC QLQ-INFO25 } & BAI & BDI-II \\
\cline { 1 - 1 } Puntuación global & $-0,12$ & $-0,13$ \\
Escalas & & \\
Información sobre enfermedad & $-0,10$ & $-0,04$ \\
Información sobre pruebas médicas & $-0,16^{*}$ & $-0,13$ \\
Información sobre tratamientos & $-0,24^{* *}$ & $-0,22^{* *}$ \\
Información sobre otros servicios & & \\
\hline Ítems individuales & & \\
Información sobre lugares de cuidado & 0,05 & $-0,03$ \\
Información pautas autocuidado & $-0,01$ & $-0,09$ \\
Información escrita & $-0,03$ & 0,01 \\
Información cd & $-0,02$ & $-0,07$ \\
Satisfacción información & $-0,25^{* *}$ & $-0,28^{* *}$ \\
Recibir más información & $0,29^{* *}$ & $0,31^{* *}$ \\
Recibir menos información & $-0,07$ & $-0,04$ \\
Utilidad de la información & $-0,21^{* *}$ & $-0,23^{* \wedge}$ \\
\hline
\end{tabular}

BAI: Inventario de Ansiedad de Beck BDI-II: Inventario de Depresión de Beck-II ${ }^{*}$ Correlación significativa en el nivel $0,05(2 \text { colas })^{* *}$ Correlación significativa en el nivel 0,01 ( 2 colas)

\section{Discusión}

Nuestros resultados, tanto de la muestra global del Inventario de Ansiedad de Beck (BAI) y del Inventario de Depresión de Beck-II (BDI-II), indican que las puntuaciones en ambos cuestionarios son bajas y la media global no indica ansiedad o depresión clínica, siendo la sintomatología depresiva más elevada y apareciendo con más frecuencia que la ansiedad.

Por otro lado, no encontramos diferencias significativas ni en la ansiedad ni en la depresión en función de las variables sociodemográficas edad, nivel de estudios, situación laboral o tener pareja ni tampoco en función de variables clínicas como el grupo de riesgo o la fase de supervivencia. Sin embargo, sí existen diferencias 
significativas, tanto en la ansiedad como en la depresión, en función del tipo de tratamiento, la calidad de vida general y específica y la información recibida. Estos resultados probablemente se deben a las características específicas de nuestra muestra ya que nuestros hombres eran en su mayoría mayores de 65 años, sin dificultades económicas (jubilados) y tenían pareja. En cuanto a las características clínicas la mayoría se encontraban en un grupo de riesgo bajo, habían sido intervenidos con tratamientos locales, en una fase de supervivencia igual o superior a 24 meses y con poca sintomatología derivada de los tratamientos o la propia enfermedad.

Con respecto a la no existencia de diferencias significativas en la ansiedad y en la depresión en función de variables sociodemográficas como la edad, el nivel académico, la situación laboral y el tener pareja, nuestros resultados se asemejan a los obtenidos por Sharpley, Bitsika y Chiristie (2013) ${ }^{(32)}$, en cuya investigación tampoco obtuvieron ninguna asociación significativa en las diferentes variables sociodemográficas en la sintomatología depresiva. Sin embargo, existen otros estudios que sí encontraron diferencias significativas en función de variables como la edad, vivir en pareja o los recursos económicos en padecer una mayor sintomatología ansiosa o depresiva ${ }^{(33-35)}$, llegando a considerar una población especialmente vulnerable a los hombres menores de 65 años, casados y con problemas económicos ${ }^{(36,37)}$. Las discrepancias entre estos estudios y nuestros resultados pueden deberse a las propias características sociodemográficas de nuestra muestra que se comentaban anteriormente.

En cuanto a las variables clínicas, coincidimos con Prasad et al. (2014) ${ }^{(34)}$, al no encontrar diferencias significativas en función de lo avanzada que esté la enfermedad en el momento del diagnóstico, aunque debemos tener en consideración que en nuestra muestra la mayoría de nuestros hombres padecían un cáncer de próstata en grupo de riesgo bajo.

Según nuestros resultados, existen diferencias significativas tanto en la ansiedad como en la depresión en función del tratamiento al que se esté sometiendo al paciente. Así, como ocurre en estudios anteriores, los hombres de nuestro estudio que están recibiendo tratamientos sistémicos padecen mayores niveles de ansiedad y/o depresión que los que son intervenidos con tratamientos locales ${ }^{(38)}$.

En consonancia con nuestros resultados, en los que no hemos encontrado diferencias significativas en la ansiedad y depresión en función de la fase de supervivencia, investigaciones previas indicaban que los hombres con cáncer de próstata suelen adaptarse adecuadamente a los tratamientos locales y no presentan un riesgo de padecer síntomas significativos de ansiedad y depresión eso sí, siempre y cuando no presenten síntomas adversos urinarios o intestinales ${ }^{(2)}$. En nuestro estudio entendemos lógicos nuestros resultados dado que la mayoría se encontraba en una fase de supervivencia igual o superior a 24 meses de un tratamiento local y con una sintomatología derivada de la enfermedad o los tratamientos baja. En caso de no ser así, es decir si existen secuelas irreversibles a raíz de los tratamientos, los estudios indican que estos hombres pueden llegar a presentar ansiedad o depresión intensa y prolongada meses o incluso años después del tratamiento ${ }^{(39)}$. En este sentido, el avance de los tratamientos, cada vez más conservadores y menos agresivos, y la existencia de fármacos o intervenciones multidisciplinares ayudan a paliar los efectos adversos facilitando por tanto la adaptación en la fase de seguimiento o supervivencia. A pesar de todo esto, tal como indicaba en sus conclusiones Watson et al. (2015), no podemos olvidar que existe un porcentaje de hombres que presentan 
ansiedad y/o depresión clínica, problemas físicos y psicosociales importantes ${ }^{(40)}, \mathrm{y}$ que el malestar emocional en estos hombres aparece con más frecuencia cuando se realizan las pruebas del PSA, en el momento de recibir la noticia, en la toma de decisiones de las diferentes opciones terapéuticas, durante el tratamiento o en la recidiva $^{(4)}$.

También coincidimos en afirmar que sigue existiendo una población especialmente vulnerable en los hombres que padecen un cáncer de próstata avanzado o en recidiva y que están recibiendo tratamientos sistémicos como los hormonales ya que estos hombres, tal como se muestra en nuestro estudio, presentan mayores niveles de ansiedad y/o depresión, además de tener menores recursos psicológicos, una mayor comorbilidad y más problemas sexuales, presentando por tanto más deteriorada la calidad de vida en general ${ }^{(10,38)}$.

Los resultados de nuestra muestra global del cuestionario de calidad de vida indican que estos hombres tienen una calidad de vida media, con una funcionalidad adecuada, siendo la función emocional la más deteriorada. Con respecto a los síntomas generales producidos por la enfermedad o por los tratamientos son bajos presentando sobre todo insomnio, estreñimiento y fatiga, el área sexual es la más afectada y tras ella, el área urinaria. Tanto los resultados de la muestra de la calidad de vida general como la específica de cáncer de próstata coinciden en su mayoría con los datos de referencia del EORTC QLQ-C30 Y EORTC QLQ-PR25 ${ }^{(27,29)}$. Los resultados indican que, al igual y como ocurría en investigaciones previas, la mayoría de las escalas de la calidad de vida se asocian significativamente con los síntomas de ansiedad o depresión. De esta forma, los hombres que padecen más síntomas de ansiedad o depresión padecen más sintomatología urinaria, intestinal, hormonal y/o sexual además de presentar más fatiga, dolor, insomnio o dificultades financieras ${ }^{(4,10)}$.

Para terminar, al evaluar la variable de la información coincidimos con los datos de referencia internacionales y nacionales en que existen escalas o ítems como los que informan sobre otros servicios (como el apoyo psicológico), los que se refieren a lugares y pautas de cuidado o dar información en soportes como $\mathrm{CD}$ son bajas e incluso deficitarias. También coincidimos en que la satisfacción y utilidad de la información es aceptable ${ }^{(30,31)}$. A pesar de que la comunicación es uno de los factores más importante en la relación médico-paciente y que conseguir transmitir una información adecuada e individualizada influye en el bienestar emocional de los pacientes, ésta es una de las tareas más difíciles, frecuentes e importantes que deben afrontar los facultativos que se dedican al campo de la oncología. A pesar de estas dificultades, no se puede obviar que el hecho de que nuestros pacientes estén más satisfechos influye en que se adapten mejor a los cambios, tengan mejor calidad de vida y padezcan menos síntomas de ansiedad o depresión $^{(41,42)}$. El hecho de que en las escalas mencionadas las puntuaciones sean tan bajas puede ser debido a la falta de disponibilidad de recursos, a la falta de formación en habilidades de comunicación o a que otros apoyos diferentes a los físicos, como el psicológico o el social, no son considerados con la importancia que se merecen. Con independencia de cuál sea el motivo, no se está facilitando que el paciente pueda optar a otros recursos o apoyos que necesitan y por lo tanto no está recibiendo la atención integral y multidisciplinar por la que tanto se aboga hoy en día. Tal como indicaba Burt et al. (2005), el facultativo debe facilitar la información necesaria, teniendo en cuenta las características de cada paciente, con información escrita o digital para minimizar el impacto de la enfermedad y 
los tratamientos ${ }^{(43)}$, más aun cuando los propios hombres admiten que tener más información les ayudarían a la hora de afrontar un diagnóstico de este tipo ${ }^{(44)}$.

La tasa de supervivencia y la edad a la que se suele detectar el cáncer de próstata pueden explicar que los trastornos mentales no se produzcan de manera tan aguda, aunque existe el riesgo de que lleguen a cronificarse y fluctúen en intensidad, dificultando su detección e infravalorando su diagnóstico. Por otro lado, estos hombres experimentan un estrés elevado, tanto de ansiedad como de depresión, desde el momento en el que el facultativo les alerta de la necesidad de descartar que padezcan un cáncer de próstata, mucho antes incluso de someterse a ningún tratamiento ${ }^{(44)}$, lo que argumenta la necesidad de que los servicios de apoyo psicosociales estén presentes mucho antes de comenzar el propio tratamiento. Es necesario investigar sobre el estado emocional de estas personas, ya que la depresión experimentada en los pacientes con cáncer de próstata aún no ha sido abordada adecuadamente y existen pocos estudios que hayan examinado la prevalencia de este trastorno, siendo extrapolable esta carencia a los trastornos de ansiedad.

En conclusión, en general los hombres que participaron en el estudio no presentan trastornos de ansiedad ni depresión y la sintomatología depresiva es más prevalente y elevada que la sintomatología relacionada con la ansiedad. Los hombres que están recibiendo tratamientos hormonales son especialmente vulnerables y tienden a presentar síntomas de ansiedad y depresión, mientras que los que han recibido tratamientos locales, en la fase de seguimiento, en general tienen buen estado emocional. Aquellos que tienen más calidad de vida o están más satisfechos con la información recibida padecen menos síntomas de ansiedad y depresión. La información debe mejorar y no sólo en aspectos como la enfermedad y los tratamientos sino también en el resto de las áreas del cuidado biopsicosocial.

\section{Referencias bibliográficas}

1. Las cifras del cáncer en España 2019 | Día Mundial del Cáncer 2019 [Internet]. [Acceso 19 de agosto de 2019]. Disponible en: https://seom.org/dmcancer/las-cifras-del-canceren-espana-2019/

2. Namiki S, Saito S, Tochigi T, Numata I, Ioritani N, Arai Y. Psychological distress in Japanese men with localized prostate cancer. Int J Urol 2007;14:924-9. doi: 10.1111/j.14422042.2007.01746.x.

3. Boonzaier A, Schubach K, Troup K, Pollard A, Aranda S, Schofield P. Development of a psychoeducational intervention for men with prostate cancer. J Psychosoc Oncol 2009;27:136-53. doi: 10.1080/07347330802616100.

4. De Sousa A, Sonavane S, Mehta J. Psychological aspects of prostate cancer: a clinical review. Prostate Cancer Prostatic Dis 2012;15:120-7. doi: 10.1038/pcan.2011.66.

5. Watts S, Leydon G, Birch B, Prescott P, Lai L, Eardley S, et al. Depression and anxiety in prostate cancer: a systematic review and meta-analysis of prevalence rates. BMJ Open 2014;4:e003901. doi: 10.1136/bmjopen-2013-003901.

6. Grassi L, Rosti G. Psychosocial morbidity and adjustment to illness among long-term cancer survivors. A six-year follow-up study. Psychosomatics 1996;37:523-32. doi: 10.1016/S0033-3182(96)71516-5.

7. Jayadevappa R, Malkowicz SB, Chhatre S, Johnson JC, Gallo JJ. The burden of depression in prostate cancer. Psychooncology 2012;21:1338-45. doi: 10.1002/pon.2032. 
8. Mohler J, Bahnson RR, Boston B, Busby JE, D'Amico A, Eastham JA, et al. NCCN clinical practice guidelines in oncology: prostate cancer. J Natl Compr Cancer Netw JNCCN 2010;8:162-200. doi:

9. National Comprehensive Cancer Network (NCCN). Prostate cancer clinical practice guidelines in oncology, version1 [Internet]. 2014. [Acceso 3 de septiembre de 2019]. Disponible en: http:/www.nccn.org/professionals/physician_gls/f_guidelines.asp.

10. Punnen S, Cowan JE, Dunn LB, Shumay DM, Carroll PR, Cooperberg MR. A longitudinal study of anxiety, depression and distress as predictors of sexual and urinary quality of life in men with prostate cancer. BJU Int 2013;112:E67-75. doi: 10.1111/bju.12209.

11. Sharp L, O’Leary E, Kinnear H, Gavin A, Drummond FJ. Cancer-related symptoms predict psychological wellbeing among prostate cancer survivors: results from the PiCTure study. Psychooncology 2016;25:282-91. doi: 10.1002/pon.3909.

12. Dąbrowska-Bender M, Słoniewski R, Religioni U, Juszczyk G, Słoniewska A, Staniszewska A. Analysis of quality of life subjective perception by patients treated for prostate cancer with the EORTC QLQ-C30 Questionnaire and QLQ-PR25 module. J Cancer Educ Off J Am Assoc Cancer Educ 2015;30. doi: 10.1007/s13187-015-0954-5.

13. Gacci M, Baldi E, Tamburrino L, Detti B, Livi L, De Nunzio C, et al. Quality of life and sexual health in the aging of pca survivors. Int J Endocrinol 2014;2014:1-16.doi: 10.1155/2014/470592.

14. Gilbert SM, Sanda MG, Dunn RL, Greenfield TK, Hembroff L, Klein E, et al. Satisfaction with Information used to choose prostate cancer treatment. J Urol 2014;191:1265-71. doi: 10.1016/j.juro.2013.12.008.

15. Oraá N, Sánchez M, Lentati G, Mendizabal E, de, Cevas FJ, Martínez N. Eficacia de las intervenciones psicológicas en hombres con cáncer de próstata. Psicooncología 2013;10: 339-51. doi: 10.5209/rev_PSIC.2013.v10.n2-3.43454.

16. van der Meulen N, Jansen J, van Dulmen S, Bensing J, van Weert J. Interventions to improve recall of medical information in cancer patients: a systematic review of the literature. Psychooncology 2008;17:857-68. doi: 10.1002/pon.1290.

17. Karnofsky DA, Abelmann WH, Craver LF, Burchenal JH. The use of the nitrogen mustards in the palliative treatment of carcinoma. With particular reference to bronchogenic carcinoma. Cancer 1948;1:634-56. doi: 10.1002/1097-0142(194811)1:4<634::AIDCNCR2820010410>3.0.CO;2-L.

18. Lobo A, Ezquerra J, Gómez Burgada F, Sala JM, Seva DïazA. El Miniexamen cognoscitivo (un test sancillo, práctico para detectar alteraciones intelectualesen pacientes medicos. Rev Psiquiatr Psicol Med 1980;14:39-57. doi:

19. Folstein MF, Folstein SE, McHugh PR. "Mini-mental state”. J Psychiatr Res 1975;12:189-98.

20. Beck AT, Steer RA. Manual. BAI. Inventario de Ansiedad de Beck (Adaptación española: Sanz J.). Madrid: Pearson; 2011.

21. Beck AT, Epstein N, Brown G, Steer RA. An inventory for measuring clinical anxiety: psychometric properties. J Consult Clin Psychol 1988;56:893-7. doi: 10.1.37//0022006x.56.6.893.

22. Sanz J. Recomendaciones para la utilización de la adaptación española del Inventario de Ansiedad de Beck (BAI) en la práctica clínica. Clin Salud 2014;25:39-48. doi: 10.5093/ cl2014a3.

23. Beck AT, Steer RA, Brown GK. Manual. BDI-II. Inventario de Depresión de Beck-II (Adaptación española: Sanz J, Vázquez C). Madrid: Pearson; 2011.

24. Aaronson NK, Ahmedzai S, Bergman B, Bullinger M, Cull A, Duez NJ, et al. The European Organization for Research and Treatment of Cancer QLQ-C30: a quality-of- 
life instrument for use in international clinical trials in oncology. J Natl Cancer Inst. 1993;85:365-76. doi: 10.1093/jnci/85.5.365.

25. Fayers P, Aaronson N, Bjordal K, Bottomley A, de Graeff A, Groenvold M, et al. EORTC QLQ-C30. Scoring manual. 3. ${ }^{\mathrm{a}}$ ed. Brussels: EORTC; 2001. 419 p.

26. Arraras JI, Arias F, Tejedor M, Pruja E, Marcos M, Martínez E, et al. The EORTC QLQ-C30 (version 3.0) Quality of Life questionnaire: validation study for Spain with head and neck cancer patients. Psychooncology 2002;11:249-56. doi: 10.1002/pon.555.

27. Arraras JI, Villafranca E, Vega FA de la, Romero P, Rico M, Vila M, et al. The EORTC Quality of Life Questionnaire for patients with prostate cancer: EORTC QLQ-PR25. Validation study for Spanish patients. Clin Transl Oncol 2009;11:160-4. doi: 10.1007/ S12094-009-0332-z.

28. Bjordal K, de Graeff A, Fayers PM, Hammerlid E, van Pottelsberghe C, Curran D, et al. A 12 country field study of the EORTC QLQ-C30 (version 3.0) and the head and neck cancer specific module (EORTC QLQ-H\&N35) in head and neck patients. EORTC Quality of Life Group. Eur J Cancer 1990 2000;36:1796-807. doi: 10.1016/s0959-8049(00)00186-6.

29. van Andel G, Bottomley A, Fosså SD, Efficace F, Coens C, Guerif S, et al. An international field study of the EORTC QLQ-PR25: a questionnaire for assessing the health-related quality of life of patients with prostate cancer. Eur J Cancer 1990 2008;44:2418-24. doi: 10.1016/j.ejca.2008.07.030.

30. Arraras JI, Greimel E, Sezer O, Chie W-C, Bergenmar M, Costantini A, et al. An international validation study of the EORTC QLQ-INFO25 questionnaire: an instrument to assess the information given to cancer patients. Eur J Cancer 1990. 2010;46:2726-38. doi: 10.1016/j.ejca.2010.06.118.

31. Arraras JI, Manterola A, Hernández B, Arias de la Vega F, Martínez M, Vila M, et al. The EORTC information questionnaire, EORTC QLQ-INFO25. Validation study for Spanish patients. Clin Transl Oncol Off Publ Fed Span Oncol Soc Natl Cancer Inst Mex. junio de 2011;13:401-10. doi: 10.1007/s12094-011-0674-1.

32. Sharpley CF, Bitsika V, Christie DRH. The incidence and causes of different subtypes of depression in prostate cancer patients: implications for cancer care. Eur J Cancer Care 2013;22:815-23.

33. Lintz K, Moynihan C, Steginga S, Norman A, Eeles R, Huddart R, et al. Prostate cancer patients' support and psychological care needs: Survey from a non-surgical oncology clinic. Psychooncology 2003;12:769-83. doi: 10.1002/pon.702.

34. Prasad SM, Eggener SE, Lipsitz SR, Irwin MR, Ganz PA, Hu JC. Effect of depression on diagnosis, treatment, and mortality of men with clinically localized prostate cancer. J Clin Oncol Off J Am Soc Clin Oncol 2014;32(23):2471-8. doi: 10.1200/JCO.2013.51.1048.

35. Weiss Wiesel TR, Nelson CJ, Tew WP, Hardt M, Mohile SG, Owusu C, et al. The relationship between age, anxiety, and depression in older adults with cancer. Psychooncology 2015;24:712-7. doi: 10.1002/pon.3638.

36. Balderson N, Towell T. The prevalence and predictors of psychological distress in men with prostate cancer who are seeking support. Br J Health Psychol 2003;8:125-34. doi: $10.1348 / 135910703321649114$.

37. Steginga SK, Occhipinti S, Dunn J, Gardiner RA, Heathcote P, Yaxley J. The supportive care needs of men with prostate cancer. Psychooncology 2001;10:66-75. doi: 10.1002/1099-1611(200101/02)10:1<66::AID-PON493>3.0.CO;2-Z.

38. Dinh KT, Reznor G, Muralidhar V, Mahal BA, Nezolosky MD, Choueiri TK, et al. Association of androgen deprivation therapy with depression in localized prostate cancer. J Clin Oncol Off J Am Soc Clin Oncol 2016;34:1905-12. doi: 10.1200/JCO.2015.64.1969. 
39. Gore JL, Kwan L, Lee SP, Reiter RE, Litwin MS. Survivorship beyond convalescence: 48-month quality-of-life outcomes after treatment for localized prostate cancer. J Natl Cancer Inst 2009;101:888-92. doi: 10.1093/jnci/djp114.

40. Watson E, Shinkins B, Frith E, Neal D, Hamdy F, Walter F, et al. Symptoms, unmet needs, psychological well-being and health status in survivors of prostate cancer: implications for redesigning follow-up. BJU Int 2016;117(6B):E10-19. doi: 10.1111/bju.13122.

41. Oraá N. Efecto de variables sociodemgráficas y clínicas en la calidad de vida y los síntomas de ansiedad y depresión en los pacientes con cáncer de próstata. [Madrid]: Universidad Complutense de Madrid; 2017.

42. Lamers RED, Cuypers M, Husson O, de Vries M, Kil PJM, Ruud Bosch JLH, et al. Patients are dissatisfied with information provision: perceived information provision and quality of life in prostate cancer patients. Psychooncology 2016;25:633-40. doi: 10.1002/ pon.3981.

43. Burt J, Caelli K, Moore K, Anderson M. Radical prostatectomy: men's experiences and postoperative needs. J Clin Nurs 2005; 14:883-90. doi: 10.1111/j.1365-2702.2005.01123.x.

44. Sharpley CF, Bitsika V, Christie DRH. "The Worst Thing Was...": Prostate cancer patients' evaluations of their diagnosis and treatment experiences. Am J Mens Health 2018;12:1503-9. doi: 10.1177/1557988318772752. 